\title{
Banco de dados VerboWeb: um panorama do léxico verbal do PB
}

\author{
Márcia Cançadoㅁ, Luana Amaral ${ }^{1}$, Letícia Meirelles ${ }^{1}$, Thaís Bechir ${ }^{1}$, Amanda \\ Oliveira $^{1}$
}
${ }^{1}$ Faculdade de Letras - Universidade Federal de Minas Gerais (UFMG) - Belo Horizonte - MG - Brasil
mcancado@ufmg.br, luanalopes@let.grad.ufmg.br, lelumeirelles@hotmail.com, thais.carvalhobechir@gmail.com, amanda_noliveira@yahoo.com.br

\begin{abstract}
In this paper, we present the proposal of description and analysis of Brazilian Portuguese verbal data present in the VerboWeb database. Verbs were grouped and classified according to the theoretical-methodological framework of Lexical Semantics, which assumes that some semantic properties of verbs determine their syntactic behavior. We show how the database is structured and the generalizations we can make about the verbal lexicon of our language.
\end{abstract}

Resumo. Neste artigo apresentamos a proposta de descrição e análise de dados verbais do português brasileiro presente no banco de dados VerboWeb. Os verbos foram agrupados e classificados de acordo com o arcabouço teórico-metodológico da Semântica Lexical, que assume que certas propriedades semânticas dos verbos determinam seu comportamento sintático. Mostramos como o banco de dados é estruturado e as generalizações que podemos fazer sobre o léxico verbal de nossa língua.

\section{Introdução}

O banco de dados VerboWeb foi criado pelas professoras doutoras Márcia Cançado, Luana Amaral e pela doutora Letícia Meirelles, no ano de 2017, tendo como ponto de partida, o projeto de descrição verbal do português brasileiro (PB) "Catálogo de Verbos do Português Brasileiro", criado e coordenado pela Profa. Dra. Márcia Cançado, que tem como principal objetivo catalogar os verbos do PB descrevendo suas propriedades sintáticas e semânticas. ${ }^{1}$ Atualmente, o desenvolvimento do banco, conta a participação de mais dois membros: a doutoranda Thaís Bechir e a estudante de mestrado Amanda Oliveira.

Neste artigo, apresentamos uma visão geral do banco de dados, mostrando nossa proposta de classificação verbal e metodologia de análise.

\section{Estrutura geral do banco de dados}

\footnotetext{
${ }^{1}$ Site do banco: http://www.letras.ufmg.br/verboweb/
} 
A proposta de classificação verbal presente no VerboWeb é norteada por uma linha de pesquisa conhecida como Semântica Lexical ou Interface Sintaxe-Semântica Lexical, que se baseia no pressuposto de que a realização sintática dos argumentos verbais é motivada pela semântica dos verbos [Fillmore 2003 [1970]; Pinker 1989; Levin 1993; Levin; Rappaport Hovav 1995, 2005; Beavers 2010; Wunderlich 2012; Cançado; Godoy; Amaral 2013, 2017; Beavers; Koontz-Garboden 2020].

Atualmente, o banco conta com 1495 verbos que estão distribuídos por 18 classes. A divisão primeira dessas classes é feita de acordo com o aspecto lexical e posteriormente, através de outras propriedades semânticas de significação. Essas propriedades semânticas são representadas em termos de papéis temáticos e através da linguagem de decomposição de predicados. Além da análise semântica, cada classe apresenta propriedades sintáticas, comuns a todos os seus membros, que são decorrentes da significação verbal. Até o presente momento, o banco conta com 43 propriedades sintáticas, exemplificadas por sentenças em cada um dos verbos que as exibem.

O VerboWeb ainda conta com um nível mais específico de classificação, que é o que chamamos de subclasses. Há um total de 7 subclasses no banco, sendo que cada uma delas está relacionada a uma classe específica. Os membros de uma subclasse apresentam as mesmas propriedades sintático-semânticas da classe, mais alguma propriedade semântica específica, que motiva a ocorrência de uma ou mais propriedades sintáticas adicionais.

Por fim, ainda há o que chamamos de propriedades não classificatórias, que são aquelas que não estão relacionadas a uma classe ou subclasse. Elas perpassam por membros de diversas classes, estando relacionadas com apenas parte da significação verbal e até mesmo com elementos pragmáticos. Até o momento, há 8 propriedades não classificatórias no banco.

Todas as classes, subclasses e propriedades são devidamente explicadas e exemplificadas no banco dados de uma maneira interativa. Para consultar essas explicações, basta clicar no conceito desejado, que é aberto um pop up com a definição do item em questão e com referências de textos teóricos sobre o assunto.

Nas próximas seções, explicaremos e exemplificaremos esses processos de classificação que utilizamos.

\section{Agrupamento pelo aspecto lexical}

Como já mencionamos, nosso primeiro nível de classificação baseia-se na noção de aspecto lexical, proposta inicialmente por Vendler (1967) e assumida posteriormente por outros autores, como Comrie (1976) e Smith (1997). De forma geral, o aspecto lexical diz respeito a como as situações descritas pelos verbos desenrolam-se no decorrer do tempo. Esse desenrolar é descrito em termos de traços aspectuais, como dinamicidade, telicidade e duratividade. A partir desses traços, Vendler (1967) propôs a existência de quatro classes aspectuais: atividades, accomplishments, achievements e estados. Os verbos de atividade descrevem situações dinâmicas, durativas e atélicas, ou seja, que não se encaminham para um resultado, ex: correr, chicotear, abraçar, etc. Verbos de accomplishment também são dinâmicos e durativos, mas, contrariamente às atividades, são télicos, ou seja, apresentam um resultado final, ex: machucar, acorrentar, abastecer, etc. Verbos de achievement são dinâmicos e télicos, como os 
accomplishments, porém majoritariamente pontuais, pois focam apenas no resultado final e não da realização de uma ação, ex: chegar, enviuvar, adormecer, etc. ${ }^{2}$ Por fim, verbos de estado não são dinâmicos, contrariamente às outras classes, são durativos e atélicos, ex: amar, existir, preocupar, etc.

Até o presente momento, no banco de dados VerboWeb, há 6 classes de verbos de atividade, 7 classes de verbos que denotam accomplishment, 2 classes de verbos que apresentam o aspecto lexical de achievement e 3 classes que denotam estados.

Tendo mostrado a classificação inicial, que é motivada pelo aspecto lexical, passemos para a descrição das diferentes classes verbais que cada classe aspectual engloba.

\subsection{Classes verbais}

As classes verbais presentes no VerboWeb são agrupamentos de verbos que apresentam, além do mesmo aspecto lexical, a mesma estrutura argumental, ou seja, o mesmo número e tipo semântico de argumentos, o que faz com que os verbos pertencentes a uma mesma classe tenham os mesmos comportamentos sintáticos. Essa classificação ocorre em um nível intermediário (medium-grained) [Levin 2010; Cançado; Amaral 2016; Cançado; Gonçalves 2016] e corresponde, em sua maioria, às classes verbais canônicas propostas na literatura em Semântica Lexical. Nesta seção, mostraremos e exemplificaremos uma classe pertencente a cada aspecto verbal.

Uma classe que faz parte das 6 classes de verbos de atividade é a que denominamos de "verbos de contato mediado pelo corpo". Ela possui 40 verbos do tipo beijar e morder, que denotam que "X age sobre $\mathrm{Y}$, por meio de um evento mediado pelo corpo" [Cançado; Amaral; Meirelles 2017]. Os verbos pertencentes a essa classe tomam dois argumentos para ter seu sentido saturado (um sujeito e um objeto), que recebem, respectivamente, os papéis temáticos de Agente e o papel que denominamos de Objeto Afetado. Além da estrutura de papéis temáticos, propomos representar o significado dos verbos dessa classe através da seguinte estrutura de decomposição de predicados: [[X ACT $<E V E N T>$ ON Y]], na qual a raiz $\langle E V E N T>$ corresponde aos eventos beijo e mordida, enquanto o predicado ON representa a ideia de contato expressa pelos verbos. Além das sentenças- bases (ex: o menino beijou a menina; o cachorro mordeu a menina), as características semânticas dos verbos licenciam os seguintes comportamentos sintáticos: (i) presença de um sintagma cognato em adjunção ( $o$ menino beijou a menina com um beijo molhado; o cachorro mordeu a menina com uma mordida forte); (ii) formação de nominalização, na qual o sujeito do verbo funciona como complemento nominal (o beijo do menino na menina; a mordida do cachorro na menina); (iii) realização da passiva eventiva (a menina foi beijada pelo menino; a menina foi mordida pelo cachorro) e; (iv) fatoração do argumento Objeto Afetado (o menino beijou a bochecha da meninal o menino beijou a menina na bochecha; o cachorro mordeu a perna da meninal o cachorro mordeu a menina na perna).

\footnotetext{
2 Cançado e Amaral (2016), baseadas em Smith (1997), afirmam que certos verbos de achievement podem apresentar uma duração, como amadurecer e derreter, porém continuam denotando eventos que falam apenas do resultado final de uma situação.
} 
Dentre as 7 classes de verbos de accomplishment, mostraremos a mais numerosa, que é a classe dos verbos de mudança de estado opcionalmente agentivos, como quebrar e machucar [Cançado; Godoy; Amaral 2013, 2017]. Fazem parte da classe 464 verbos, que denotam que " $\mathrm{X}$ age, causando $\mathrm{Y}$ ficar em um determinado estado" [Cançado; Amaral; Meirelles 2017]. Os verbos dessa classe tomam dois argumentos para ter seu sentido completo, sendo que o argumento que ocupa a posição de sujeito pode receber os papéis temáticos de Causa ou de Agente (a queda quebrou o vasol o menino machucou o colega intencionalmente), enquanto o argumento que ocupa a posição de objeto recebe o papel temático de Paciente. A estrutura de decomposição de predicados que representa o sentido da classe é a seguinte: [[X ACT (Volition)] CAUSE [BECOME [Y <RESULT-STATE $>]]$. O predicado VOLITION, entre parênteses, representa a opcionalidade da agentividade na realização do evento, enquanto a associação dos predicados CAUSE e BECOME representam a causação de uma mudança, mais especificamente uma mudança que culmina em um estado resultante, representado pela raiz <RESULT-STATE >, correspondente aos estados quebrado e machucado. As propriedades semânticas da classe licenciam os seguintes comportamentos sintáticos: (i) participação na alternância causativo-incoativa (a queda quebrou o vasol o vaso (se) quebrou; o acidente machucou a meninal a menina (se) machuсоu); (ii) adjunção da causa na forma incoativa (o vaso (se) quebrou com a queda; a menina (se) machucou com o acidente); (iii) realização das formas passivas eventiva, resultativa e estativa (o vaso foi/ficou/está quebrado; a menina foi/ficou/está machucada).

Passando para os achievements, esse aspecto lexical também apresenta uma classe de mudança de estado, formada por 85 verbos do tipo amadurecer e azedar. Esses verbos denotam que "X passa a ficar em um determinado estado" [Cançado; Amaral; Meirelles 2017] e apresentam a seguinte estrutura de decomposição de predicados, que é a contraparte da dos verbos de mudança de estado causativos que apresentamos anteriormente: [BECOME [X $<R E S U L T-S T A T E>]]$. Essa estrutura evidencia que os verbos da classe falam apenas de um estado final resultante, que corresponde aos estados de ficar maduro e ficar azedo. Os verbos da classe tomam apenas um argumento para ter seu sentido saturado e esse argumento recebe o papel temático de Paciente. As propriedades semânticas licenciam os seguintes comportamentos sintáticos: (i) participação na alternância incoativa-causativa ${ }^{3}$ ( calor amadureceu a banana; o excesso de calor azedou o leite); (ii) adjunção de causa indireta (a banana amadureceu com o calor; o leite azedou com o excesso de calor); (iii) realização das formas passivas resultativa e estativa (a banana ficou/está madura; $o$ leite ficou/está azedo) e; (iv) não aceitação do clítico se (*a banana se amadureceu; *o leite se azedou).

Por fim, dentre as classes de estado, mostraremos a classe dos verbos de estado psicológico do tipo amar. Compõem a classe 34 verbos que denotam que "X está em determinado estado psicológico em relação a algo ou a alguém" [Cançado; Amaral; Meirelles 2017]. Os verbos tomam dois argumentos para ter seu sentido completo, sendo um sujeito Experienciador e um objeto que recebe o papel temático de Objeto Estativo. A estrutura de decomposição de predicados da classe é [X $\langle$ PSYCH-STATE $>$ Y], na qual a categoria ontológica $\langle P S Y C H-S T A T E>$ é subespecificada como um estado

\footnotetext{
${ }^{3}$ Cançado e Amaral (2016), baseadas em Haspelmath (1993), propõem que os verbos dessa classe são basicamente incoativos, pois não aceitam o clítico se, que seria um marcador de alternância no PB.
} 
psicológico que, portanto, relaciona dois indivíduos, no sentido amplo do termo. As propriedades sintáticas derivadas dessas características semânticas são: (i) os verbos licenciam a fatoração do argumento Objeto Estativo (o rapaz ama o jeito meigo da namoradal o rapaz ama a namorada pelo seu jeito meigo; a moça repudiou as maneiras do rapaz/ A moça repudiou o rapaz por suas maneiras) e; (ii) licenciam uma perspectiva passiva (a namorada é amada pelo rapaz; o rapaz foi repudiado pela moça).

Tendo descrito uma classe pertencente a cada uma das classes aspectuais presentes no VerboWeb, descreveremos a seguir uma das subclasses do banco de dados.

\subsubsection{Subclasses}

O conceito de subclasses está relacionado a um nível mais fino de classificação semântica, conhecido como fine-grained [Levin 2010; Cançado; Amaral 2016; Cançado; Gonçalves 2016], pois diz respeito a propriedades que não estão presentes na estrutura argumental dos verbos, mas fazem parte do seu sentido idiossincrático. Segundo Cançado, Amaral e Meirelles $(2017,2018)$, cada subclasse é relacionada a uma classe verbal no nível medium-grained e os verbos que compõem as subclasses, apresentam, além das propriedades sintático-semânticas da classe a qual pertencem, pelo menos uma propriedade sintática a mais, que é decorrente do seu significado específico.

Um exemplo de subclasse é a dos verbos com objeto recíproco. Segundo autores como Dixon (1992), Siloni (2007), Godoy (2008), Bechir (2016), verbos recíprocos são aqueles que exigem que um dos seus argumentos denote dois (ou mais) referentes no mundo, como o verbo afastar na sentença a faxineira afastou os móveis. No nível medium-grained, o verbo afastar pertence à classe dos verbos de mudança de estado opcionalmente agentivos, pois apresenta todas as propriedades dos membros da classe: (i) aceita uma Causa ou um Agente como sujeito (o tremor de terra afastou os móveis/ a faxineira afastou os móveis intencionalmente); (ii) licencia a forma incoativa com o clítico se (os móveis (se) afastaram); (iii) licencia a causa em adjunção na forma incoativa (os móveis (se) afastaram com o tremor de terra) e; (iv) licencia as formas passivas eventiva, resultativa e estativa (os móveis foram/ficaram/estão afastados).

Contudo, por ter um argumento que denota dois ou mais referentes (os móveis), o verbo afastar apresenta os seguintes comportamentos sintáticos específicos: (i) licencia a forma descontínua [Godoy 2008] desse argumento (a faxineira afastou um móvel do outro); (ii) licencia a versão descontínua da forma incoativa (um móvel (se) afastou do outro) e; (iii) licencia a versão descontínua das formas passivas eventiva, resultativa e estativa (um móvel foi/ficou/está afastado do outro). Fazem parte da subclasse de verbos com objeto recíproco 33 verbos como afastar, juntar, mesclar, separar, etc.

Até o presente momento, há 7 subclasses no VerboWeb, sendo elas: verbos com objeto recíproco (ex: afastar), verbos com sujeito recíproco (ex: conversar, brigar), verbos de modo de fala (ex: berrar, urrar); verbos instrumentais com dois instrumentos (ex: balear, flechar), verbos de remoção (ex: lavar, limpar), verbos de criação de imagem (ex: bordar, carimbar) e verbos de contato (achatar e amassar). Cada uma dessas subclasses está ligada a uma propriedade sintática decorrente do sentido específicos dos verbos que as compõem. 
Tendo mostrado e exemplificado o conceito de subclasse que utilizamos no banco, passemos para a explicação daquilo que chamamos de propriedades não classificatórias.

\subsection{Propriedades não classificatórias}

Além das propriedades sintáticas e semânticas que compõem as classes e subclasses, há alguns comportamentos sintáticos que são decorrentes de propriedades semânticas (e, às vezes, pragmáticas) mais gerais, perpassando por várias classes verbais. Esse tipo de propriedade sintática é chamado de propriedade não classificatória e tem como um de seus exemplos a reflexivização [Camacho 2003 Doron; Rappaport Hovav 2009; Godoy 2012, dentre outros].

A reflexivização está ligada ao conceito de voz reflexiva trazido nas gramáticas tradicionais e basicamente ocorre com verbos transitivos que aceitam um Agente na posição de sujeito. No $\mathrm{PB}$, o pronome reflexivo se substitui o argumento em posição de objeto, passando a ter a mesma denotação que o sujeito, como em a menina se lavou. Segundo Godoy (2012), o pronome se pode ser substituído pela forma ele(a) mesmo(a): a menina lavou ela mesma. No banco de dados VerboWeb, 269 dos 1495 verbos analisados realizam a reflexivização, e esses verbos pertencem a várias classes distintas: o menino se beijou (verbos de contato mediado pelo corpo); a menina se ama (verbos de estado psicológico); o menino se machucou para não ficar de castigo (verbos de mudança de estado opcionalmente agentivos).

Há 8 propriedades não classificatórias no VerboWeb até o momento: alternância agente-beneficário (a menina cortou o cabelo com um ótimo cabeleireiro), alternância condutor-veículo (a moça parou a Mercedes na garagem/ a Mercedes parou na garagem), alternância incoativa periférica (a roupa já lavou); alternância parte-todo (o João quebrou o braço), reflexiva média (a menina se enfiou no armário); reflexiva média da forma descontínua (uma menina se afastou da outra para dançar); reflexivização (a menina se lavou) e um tipo de alternância aspectual de verbos de accomplishment para estados com processo de intransitivização (vidro de azeitona não abre fácil).

Tendo mostrado as propriedades não classificatórias presentes no banco de dados, finalizaremos a descrição do funcionamento do VerboWeb com algumas breves generalizações sobre o PB.

\section{Considerações finais}

Como já mencionamos neste artigo, o banco de dados VerboWeb é fruto de um extenso trabalho de pesquisa idealizado e coordenado pela Professora Dra. Márcia Cançado e desenvolvido pelos membros do Núcleo de Pesquisa em Semântica Lexical (NuPeS) da Faculdade de Letras da Universidade Federal de Minas Gerais. Grande parte dos dados e análises que estão presentes no banco é baseada em trabalhos desenvolvidos por membros do grupo desde sua criação até o momento atual.

Ao colocarmos e adaptarmos as análises ao modelo do banco, foi possível perceber algumas generalizações em nossa língua. Por exemplo, 800 verbos do VerboWeb denotam accomplishments, sendo consequentemente eventos causativos, enquanto os demais 695 verbos distribuem-se pelas classes de atividade, achievement e 
estado. Esse padrão reflete a afirmação de que a causação é uma das noções mais básicas presentes em nossa cognição [Langacker 1990; Croft 1991].

Também pudemos notar que a noção de contato é relevante para a classificação verbal. Observamos que os verbos que denotam atividades são divididos em classes de acordo com a presença ou ausência de contato físico entre os indivíduos envolvidos no evento, e no fato de esse contato ser mediado pelo corpo (verbos do tipo abraçar), por um instrumento externo ao verbo (verbos do tipo lavar) ou por um instrumento incorporado ao nome do verbo (verbos do tipo chicotear e patinar).

Além disso, ainda pudemos perceber que outras propriedades, como os verbos denotarem uma eventualidade psicológica, expressarem uma relação de posse ou de locação, também são propriedades semânticas que têm impacto na sintaxe, pois temos algumas classes agrupadas a partir dessas propriedades: (i) eventualidade psicológica verbos de estado psicológico do tipo amar e do tipo preocupar; (ii) relação de posse verbos de locatum (ex: acorrentar), verbos de mudança de estado de posse (ex: abastecer) e; (iii) relação de locação - verbos de location (ex: ensacar), verbos de mudança de estado locativo (ex: abrigar), verbos de achievement que denotam mudança de lugar (ex: chegar) e verbos de estado que expressam a ideia de existência (ex: existir e haver).

Por fim, gostaríamos de ressaltar que, embora o banco e as análises nele presentes tenham sido desenvolvidas baseadas no arcabouço teórico-metodológico da Semântica Lexical, os dados e as propriedades do VerboWeb podem ser utilizados por pesquisadores de outras linhas de pesquisa que estejam interessados na análise do PB. Além disso, professores dos ensinos fundamental e médio, que desejam aprender mais sobre a semântica e a sintaxe verbal, também podem se valer do banco como uma maneira de facilitar o ensino de nossa língua, já que abordagens linguísticas têm estado cada vez mais presentes nas salas de aula. Por fim, pelo fato de fornecer um extenso panorama do funcionamento do léxico verbal do $\mathrm{PB}$, o banco talvez possa ser útil para a checagem de dados de tradução, já que apresenta uma série de possibilidades de ocorrências sintáticas de nossa língua.

\section{Referências}

Beavers, J. (2010). The Structure of Lexical Meaning: Why Semantics Really Matters. Language. 86, p. 821-864.

Beavers, J.; Koontz-Garboden, A. (2020), The Roots of Verbal Meaning. Oxford: Oxford University Press.

Bechir, T. (2016). Os verbos recíprocos intransitivos no português brasileiro. Monografia de Bacharelado em Português (Estudos Linguísticos). Faculdade de Letras, UFMG, Belo Horizonte, Brasil.

Cançado, M., \& Amaral, L. (2016). Introdução à Semântica Lexical: Papéis Temáticos, aspecto lexical e decomposição de predicados. Editora Vozes, Petrópolis, RJ, Brasil.

Cançado, M, Amaral, L., \& Meirelles, L. (2017). VerboWeb: classificação sintáticosemântica dos verbos do português brasileiro. Banco de dados lexicais. UFMG. Disponível em: http://www.letras.ufmg.br/verboweb. 
Cançado, M., Amaral, L., \& Meirelles, L. (2018). VerboWeb: uma proposta de classificação verbal. Revista da Anpoll, n. 46, v. 1.

Cançado, M., Godoy, L., \& Amaral, L. (2013). Catálogo de verbos do português brasileiro: classificação verbal segundo a decomposição de predicados. Vol I. Verbos de mudança, 1 ed. Editora UFMG, Belo Horizonte, Brasil.

Cançado, M., Godoy, L., \& Amaral, L. (2017). Catálogo de verbos do português brasileiro: classificação verbal segundo a decomposição de predicados. Vol I. Verbos de mudança, 2 ed. Edição Revisada Amazon. 2017.

Cançado, M., \& Gonçalves, A. (2016). Lexical Semantics: verb classes and alternations. In L. Wetzels, S. Menuzzi, \& J. Costa (Eds.), The Handbook of Portuguese Linguistics. Willey/Blackwell, 374-391.

Camacho, R. (2003). Em defesa da categoria de voz média no português. DELTA, v. 19, n.1, p. 91-122.

Comrie, B. (1976), Aspect: an Introduction to the study of verbal aspect and related problems. Cambridge: Cambridge University Press.

Croft, W. (1991), Syntactic Categories and Grammatical Relations. Chicago: University of Chicago Press.

Dixon, R. (1992), A new approach to English grammar, on semantic principles. Oxford: Clarendon Press.

Doron, E.; Rapapport Hovav, M. (2009) "A unified approach to reflexivization in Semitic and Romance". In: Bendjaballah et al. (orgs.) Brill's Annual of Afroasiatic Languages and Linguistics 1. Leiden: Brill, p. 75-105.

Godoy, L. (2008), Os verbos recíprocos no PB: interface sintaxe-semântica lexical. (Mestrado em Estudos Linguísticos) - Faculdade de Letras, Universidade Federal de Minas Gerais, Brasil.

Godoy, L. (2012), A reflexivização no português brasileiro e a decomposição semântica de predicados. Tese (Doutorado em Estudos Linguísticos) - Faculdade de Letras, UFMG, Belo Horizonte.

Fillmore, C. (2003 [1970]) "The grammar of hitting and breaking". In: Fillmore (ed.), Form and meaning in language: Papers on semantic roles, p. 123-139. Stanford: CSLI Publications

Haspelmath, M. (1993) "More on typology of inchoative/causative verb alternations". In: Comrie \& Polinsky. Causatives and transitivity. Amsterdam: John Benjamins, p. $87-120$.

Langacker, R. (1990), Concept, Image and Symbol: The Cognitive Basis of Grammar. Berlin: Mouton de Gruyter.

Levin, B. (1993), English verb classes and alternations: A preliminary investigation. Chicago: University of Chicago Press.

Levin, B. (2010) "What is the best grain- size for defining verb classes?" Conference on Word Classes: nature, Typology, Computational Representations, Second TRiPLE international Conference, Università Roma Tre, Rome, March 24-26. 
Levin, B.; Rappaport Hovav, M. (1995), Unaccusativity: At the syntax-lexical semantics interface. Cambridge: The MIT Press.

Levin, B.; Rappaport Hovav, M. (2005), Argument realization. Cambridge: Cambridge University Press.

Pinker, S. (1989), Learnability and Cognition: The Acquisition of Argument Structure. Cambridge, MA: MIT Press.

Siloni, T. (2007) "The syntax of reciprocal verbs: an overview". In: König and Gast (eds.) Reciprocals and reflexives: cross-linguistics and theoretical explorations. Berlin: Mouton de Gruyter.

Smith, C. (1997), The Parameter of Aspect. Dordrecht: Kluwer.

Vendler, Z. (1967), Linguistics in philosophy. Ithaca: Cornell.

Wunderlich, D. (2012) “Lexical Decomposition in Grammar". In: Werning, Hinzen and Machery (Eds.). The Oxford Handbook of Compositionality. Oxford: Oxford University Press, p. 307-327. 